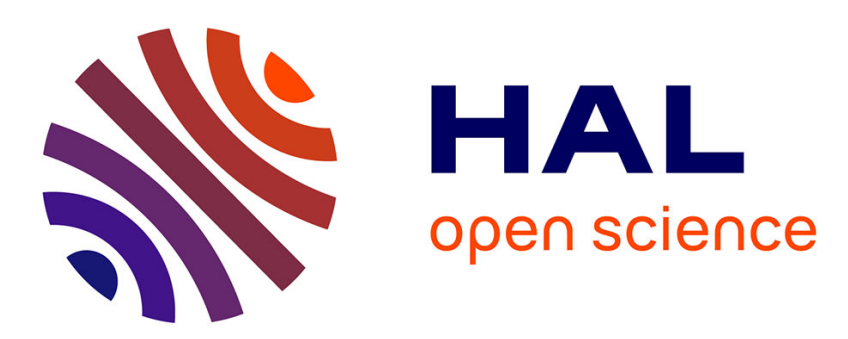

\title{
Takama, Debeinnu, âli et les autres... ou la possible histoire d'un groupe tributaire de l'Ahaggar : les Dag- âli Paul Pandolfi
}

\section{To cite this version:}

Paul Pandolfi. Takama, Debeinnu, âli et les autres... ou la possible histoire d'un groupe tributaire de l'Ahaggar: les Dag- âli. Journal des Africanistes, 1996, 66, pp.203 - 224. 10.3406/jafr.1996.1101 . halshs-03175279

\section{HAL Id: halshs-03175279 \\ https://shs.hal.science/halshs-03175279}

Submitted on 19 Mar 2021

HAL is a multi-disciplinary open access archive for the deposit and dissemination of scientific research documents, whether they are published or not. The documents may come from teaching and research institutions in France or abroad, or from public or private research centers.
L'archive ouverte pluridisciplinaire HAL, est destinée au dépôt et à la diffusion de documents scientifiques de niveau recherche, publiés ou non, émanant des établissements d'enseignement et de recherche français ou étrangers, des laboratoires publics ou privés. 


\section{Takama, Debeinnu, âli et les autres... ou la possible histoire d'un groupe tributaire de l'Ahaggar : les Dag-âli}

In: Journal des africanistes. 1996, tome 66 fascicule 1-2. pp. 203-224.

\section{Résumé}

Les Dag-yâli forment la plus importante des tribus roturières de l'Ahaggar et l'ethnographie les a un peu négligés au profit de la tribu noble des Kel-yela. L'auteur rappelle et commente les traditions d'origine de la tribu, avant d'aborder l'évolution plus récente des groupes qui la composent. II fait apparaître qu'elle semble composée, d'une part de lignages très anciennement installés dans la région et d'autre part, de lignages plus récents au sein desquels se recrute le détenteur de la chefferie.

\section{Abstract}

The Dag-yâli are the most important common tribe of Ahaggar and ethnography rather neglected them prefering to study the noble tribe of the Kel-yela. The author mentions and comments the traditions concerning the origin of the tribe, before considering the latest evolution about the groups that compose it. It appears that this tribe seems composed, on the one hand, of lineages settled formely in the region, and on the other hand of more recent lineages within which is recuited the chieftain.

Citer ce document / Cite this document :

Pandolfi Paul. Takama, Debeinnu, âli et les autres.. ou la possible histoire d'un groupe tributaire de l'Ahaggar : les Dag-âli. In: Journal des africanistes. 1996, tome 66 fascicule 1-2. pp. 203-224.

doi : 10.3406/jafr.1996.1101

http://www.persee.fr/web/revues/home/prescript/article/jafr_0399-0346_1996_num_66_1_1101 


\section{Takama, Debeinnu, $\gamma a ̂$ li et les autres... ou la possible histoire d'un groupe tributaire de l'Ahaggar: les Dag-Yâli.}

\section{Résumé}

Les Dag- $\gamma a ̂ l i$ forment la plus importante des tribus roturières de l'Ahaggar et l'ethnographie les a un peu négligés au profit de la tribu noble des Kel- $\gamma$ ela. L'auteur rappelle et commente les traditions d'origine de la tribu, avant d'aborder l'évolution plus récente des groupes qui la composent. Il fait apparaître qu'elle semble composée, d'une part de lignages très anciennement installés dans la région et d'autre part, de lignages plus récents au sein desquels se recrute le détenteur de la chefferie.

Mots-clefs

Touaregs, Ahaggar, alliance, parenté, mariage.

\section{Abstract}

The Dag- $\gamma a$ ali are the most important common tribe of Ahaggar and ethnography

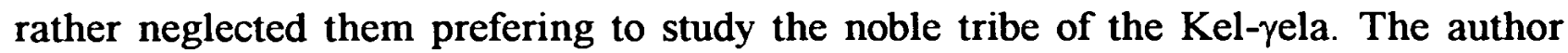
mentions and comments the traditions concerning the origin of the tribe, before considering the latest evolution about the groups that compose it. It appears that this tribe seems composed, on the one hand, of lineages settled formely in the region, and on the other hand of more recent lineages within which is recuited the chieftain.

Keywords

Touaregs, Ahaggar, union, kinship, marriage.

\footnotetext{
* Centre d'Antropologie Généralisée, Université de La Réunion.
} 
La confédération des Touaregs Kel-Ahaggar, qui vivent dans le massif de l'Ahaggar et ses alentours (Sud algérien), combine tout à la fois une structure hiérarchique et nombre de caractéristiques propres aux sociétés de type segmentaire.

D'une part, la société est divisée en strates hiérarchiques ou "rangs" pour reprendre une expression de P. Bonte (1986:4). Soit de haut en bas de l'échelle sociale : les aristocrates (ihaggâren), les tributaires (imrad ou Kel-Ulli) et les esclaves (iklan). Plus, depuis la seconde moitié du XIXème, des cultivateurs sédentaires (izeggayen) originaires du Touat-Tidikelt mais venus s'installer dans l'Ahaggar pour y cultiver les terrasses alluviales. Dans la société traditionnelle, ces différentes strates sociales étaient regroupées au sein d'une unité politique (ettebel) à la tête de laquelle se trouvait un chef, dénommé amenukal, choisi parmi les aristocrates bénéficiant d'un droit d'accès au pouvoir transmis par voie matrilinéaire. La subordination politico-économique des Kel-Ulli se marquait notamment dans le payement par les tributaires d'un impôt en nature (tiwsé) à l'amenukal.

D'autre part, les Kel-Ahaggar se répartissent en groupes de descendance (tawsit) à forte tendance endogame - surtout chez les Kel-Ulli - et dont les membres se réclament d'un ancêtre commun. La plupart de ces tawsit se subdivisent en plusieurs sections dont les noms font souvent référence à la toponymie des territoires habituellement dévolus à ces groupes.

Les principales caractéristiques de cette société sont inscrites dans les récits d'origine propres aux Kel-Ahaggar. Ces récits mettent en scène deux ancêtres féminins mythiques : Tin-Hinan et Takama. Nous en possédons plusieurs versions; les différences (plus ou moins importantes) existant entre elles sont toutes éminement signifiantes'. Dans le cadre de cet article nous nous en tiendrons cependant au fond commun qui leur est propre. Dans tous les cas, il s'agit d'ancêtres féminines : Tin Hinan et Takama sont présentées comme deux femmes extérieures. Dans les versions les plus développées, il est précisé que ce sont des femmes Bêraber originaires du Tafilalet. Arrivées dans l'Ahaggar, elles s'installèrent dans la région de Silet. Le pays, selon certaines versions, était alors quasiment vide. Seuls y résidaient, dans la partie montagneuse la plus élevée (monts Taessa) quelques membres des Isabaten, restes d'une population autrefois plus nombreuse mais qui avait été en grande partie exterminée lors d'une expédition guerrière menée par les Sehbaba ${ }^{2}$ au Sahara central.

\footnotetext{
${ }^{1}$ Nous avons commenté et analysé les différentes versions de ces récits d'origine propres aux Kel-Ahaggar dans notre thèse d'ethnologie (Pandolfi 1995b). Cette analyse permet de cerner les enjeux politiques qui fondent les différences repérables d'une version à l'autre.

${ }^{2}$ Ces envahisseurs qui, dès le VIIlème siècle semble-t-il, ont tenté de convertir les Isabaten à l'Islam sont aussi dénommés Anbiya (ou Lambiya). Voir la notice fort documentée que leur a consacré M. Gast dans l'Encyclopédie Berbère (1988 : 635-636).
} 
Concernant ces Isabaten, G. Camps (1986:281) résume ainsi les recherches préhistoriques les plus récentes :

"on est tenté de voir dans ces Isebeten des populations berbères préislamiques, très proches des Garamantes. Leur nom même n'est pas inconnu des historiens puisque un peuple de Cyrénaique et sans doute du Sahara voisin, portait le nom d'Asbytes (Hérodote IV, 170) ; ces Libyens passaient pour d'excellents conducteurs de char. Nous verrions volontiers dans ces Isebeten les descendants des "Equidiens" auteurs des gravures et des peintures de style caballin et des plus anciens tifinar."

Des récits d'origine comme des diverses anecdotes qui circulent encore à l'heure actuelle à propos des Isabaten deux traits récurrents se dégagent :

- Les Isabaten sont toujours présentés comme les plus anciens occupants connus de l'Ahaggar. Ils résidaient là bien avant l'arrivée de Tin-Hinan et Takama.

- L'image qui est donnée des Isabaten est toujours fortement dévalorisée.

Dans la version la plus complète que nous ayons du mythe de Tin-Hinan (Foucauld 1951:533) il est précisé que ces "idolâtres" étaient "courts d'esprit " et "parlaient la langue touarègue mais en un dialecte spécial et grossier". Les récits

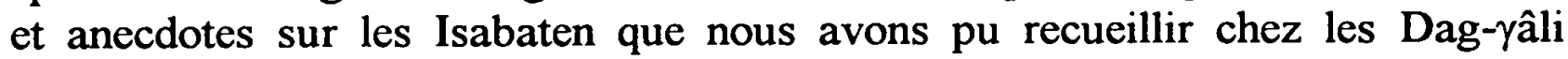
confirment tous un tel portrait. Habitant la région de la Taessa, zone montagneuse située au coeur même de l'Atakor, ils portaient des vêtements de peau $^{3}$, ignoraient la tente et tout autre type d'habitation et vivaient dans les très nombreuses grottes que l'on trouve en ce lieu. Outre leurs troupeaux de chèvres, ils assuraient leur subsistance grâce à la cueillette et surtout la chasse. L'insistance sur la bêtise, l'esprit borné des Isabaten est fortement marquée dans ces récits qui mettent au premier plan nombre d'attributs propres à l'image stéréotypée du Touareg. Les Isabaten sont ici la contre-image négative du Touareg. La stupidité dont ils font preuve les classe tout à la fois comme Isabaten et comme non-touareg dans la mesure où elle se manifeste essentiellement par leur incapacité à maîtriser les éléments les plus fondamentaux de la culture touarègue. Définition "en creux" en quelque sorte : non contents de ne point connaitre la tente et de vivre dans des grottes, ils ignorent aussi l'animal par excellence qu'est le chameau. Quand ils parlent c'est en un tamâhaq grossier, incorrect et incompréhensible pour les Dag- $\gamma a ̂ l i$ actuels. Et les vêtements (voile de tête, sandales de type igatimen...) les plus prisés par les touaregs Kel-Ahaggar ne sont pas mieux traités ${ }^{4}$. Ainsi les traits les plus

\footnotetext{
${ }^{3}$ Ces vêtements de peau étaient encore portés par certains Kel-Ahaggar au début de ce siècle comme l'a relevé Lhote, dans un article avec photos, sur ce sujet (Lhote 1979). Selon G. Tillion

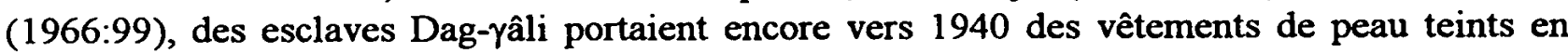
rouge et garnis de franges.

${ }^{4}$ Comme le montrent certains récits qui mettent en scène des Isabaten ignorant tout des igatimen ou du tissu aleccu (cf. Pandolfi 1995b).
} 
souvent utilisés pour caractériser la culture touarègue, pour construire le stéréotype du Touareg (tente, chameau, maîtrise et beauté de la langue, voile...) sont étrangers aux Isabaten. Voilà pour la légende. Venons-en aux faits actuels, dont nous verrons qu'ils ne sont pas sans rapport avec la légende.

La distinction hiérarchique aristocrates/tributaires est quant à elle systématiquement justifiée dans ces récits d'origine. Takama, de qui sont censés descendre les plus anciens et plus importants groupes tributaires de l'Ahaggar (Dag- $\gamma$ ali, Ayt-Lowayen et Kel-Ahnet), est présentée soit comme la servante soit comme la soeur cadette de Tin-Hinan, ancêtre présumée elle de tous les aristocrates ou, selon la version de référence, des seuls Kel-gela.

Jusqu'à présent l'attention des ethnologues et autres spécialistes des KelAhaggar s'était essentiellement - voire exclusivement - portée sur Tin Hinan et sur la reconstitution de l'histoire du groupe aristocratique dominant des Kel-yela. En tentant d'adopter un autre point de vue, en focalisant notre étude et sur Takama et sur le groupe tributaire des Dag- $\gamma$ âli nous nous sommes très rapidement heurté à de nombreuses difficultés. La première d'entre elles concernait la segmentation même de cette tawsit. Si en effet l'on se réfère sur ce sujet aux écrits de nos prédécesseurs la plus grande confusion semble régner.

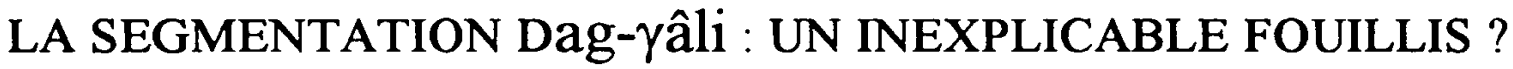

Selon Benhazera, la tawsit Dag-yali se décomposerait en trois sousgroupes : Imesîliten, Dag- $\gamma a ̂$ li et Ulad-Irdal auxquels s'ajouteraient également les Ikeccemaden "pouvant être considérés comme faisant partie intégrante des DagR'ali car de nombreuses alliances les unissent." (1908:143).

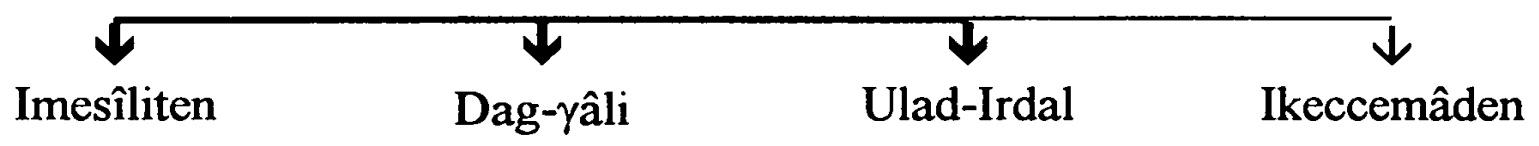

Quelque vingt ans plus tard, F. Demoulin (1926) estime lui que la tawsit

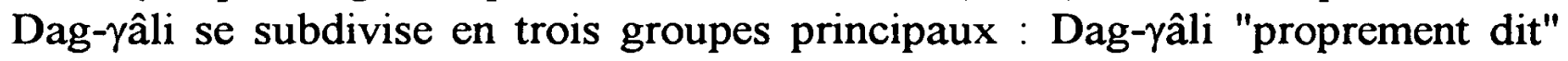
(eux-mêmes scindés en Kel-Tamanrasset et Kel-Terhenânet), Imesîliten et Ikeccemaden ${ }^{5}$.

\footnotetext{
${ }^{5}$ Pour éviter des confusions possibles entre deux réalités désignées par le même terme, nous avons adopté, dans la suite de notre exposé, les conventions typographiques suivantes: Dag- $\gamma a l i$ désigne la tawsit elle même alors que "Dag- $\gamma a l i "$ renvoie à la seule "moitié" composée des KelTerhenânet et Kel-Tamanrasset. "Moitié" nommée par certains auteurs "Dag- $\gamma$ ali proprement dit".
} 


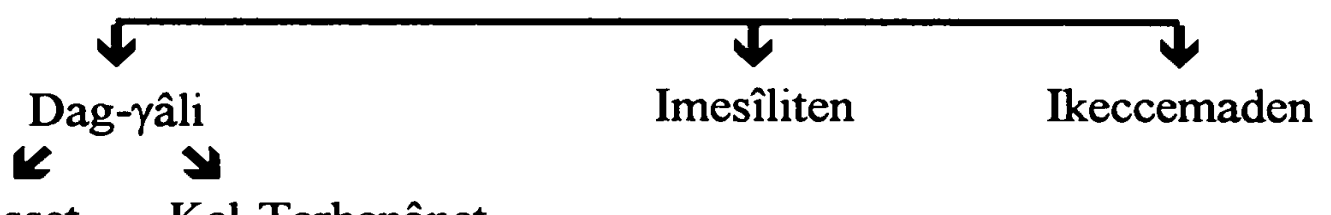

Kel-Tamanrasset Kel-Terhenânet

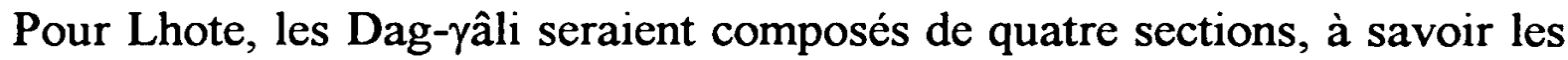
Imesîliten, Ikeccemaden, Ulad-Irdal et Dag-Mertemek. A ces quatre divisions principales viendraient s'ajouter "quelques descendants de la tribu légendaire des Isabaten " (1984:72).

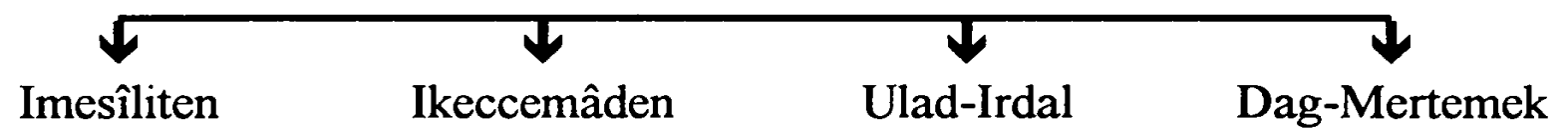

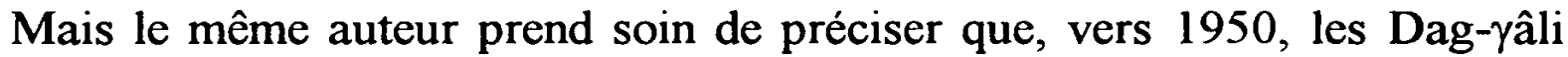
"se divisent en : Kel-Tahat, Kel-Tamanrasset, Kel-Terhenânet, Kel-Hêrafek, Kel-Arrechoum, divisions correspondant aux régions qu'ils habitent. " (idem:73).

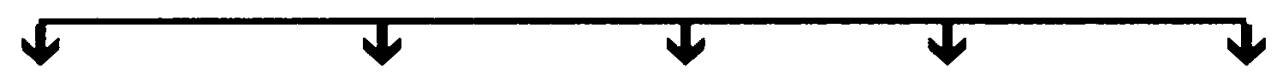

Kel-Tamanrasset Kel-Terhenânet Kel-Hêrafek Kel-Tahat Kel-Arrechoum

Au début des années 1960, P. Rognon (1962:180) propose comme sections Dag-yâli les cinq groupes suivants: Kel-Tamanrasset/Imesîliten / KelTerhenânet / Kel-Tesaylit / Kel-Hirafok.

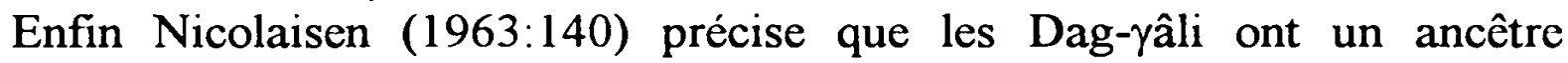
masculin éponyme ( $\gamma a \hat{l i}$ ) qui vivait il y a sept générations. Ils seraient, selon le

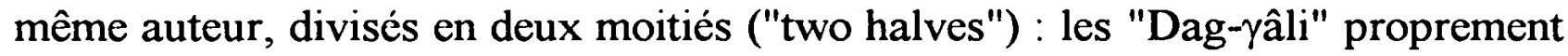
dits et les Imesîliten réparties en quatre sections: Kel-Tamanrasset, KelTerhenânet, Kel-Hirafok et Kel-Tinhart (Idem:148). Les deux premières

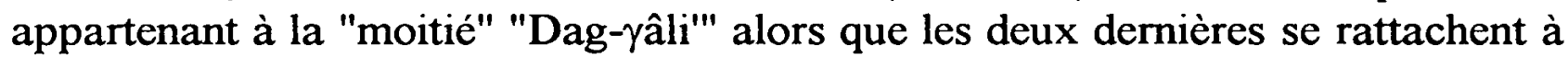
la "moitié" Imesîliten.

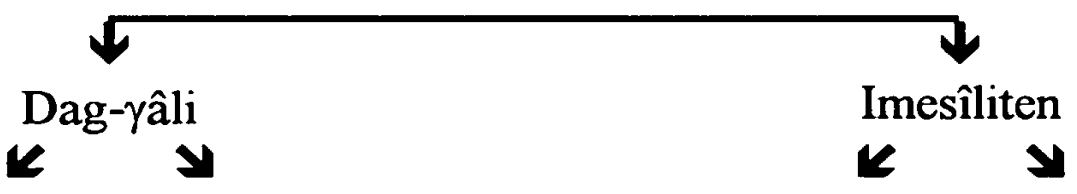

Kel-Tamanrasset Kel-Terhenânet Kel-Hirafok Kel-Tinhart

Ainsi, au vu des découpages proposés par les auteurs ci-dessus, la plus grande confusion semble régner rendant difficile l'établissement d'une nomenclature stable. C'est là un problème qui n'est pas spécifique à l'Ahaggar. S. Bernus s'est heurtée à une difficulté similaire quand elle a comparé les listes des groupes recensés, au milieu du XIXème siècle, par l'explorateur allemand Barth aux nomenclatures plus récentes des Iwllemmeden et des touaregs de l'Aïr 
$(1976: 105)^{6} . C^{\prime}$ est également une question à laquelle s'est trouvée confrontée l'administration coloniale. La lecture des "Tableaux de l'organisation des tribus" (A.O.M $8 \mathrm{HH}$ ), établis annuellement depuis 1925, est à cet égard significative. Ces documents recensaient les différentes tawsit de l'Ahaggar ainsi que leurs subdivisions et indiquaient pour chacun de ces niveaux de segmentation les noms de leurs représentants (ampar). Mais, d'une année sur l'autre, tant le nombre que la dénomination de ces divisions (notamment dans le cas des Dag- $\gamma a ̂ l i) ~ s e$ modifient. Le rédacteur du "Tableau" de l'année 1929 ajoutera d'ailleurs une note introductive dans laquelle perce son désarroi :

"Quand la nouvelle annexe du Hoggar aura été créée, toute cette organisation sera à étudier plus en détails, car il y a de nombreuses discordances entre les études déjà faites à ce sujet ; et les Touaregs euxmêmes ne semblent pas toujours très fixés sur les rapports des sections et sous-sections les unes avec les autres. /.../ en réalité, tout cela est assez compliqué et cadre mal avec les exigences de notre esprit méthodique. Il est donc assez difficile de présenter l'organisation des Touaregs sous forme de tableau et cependant, sans tableau, il est complètement impossible de s'y reconnaître."

Perplexité à laquelle font écho, dès 1903, ces lignes du capitaine Cauvet alors chef de l'Annexe d'In-Salah :

"...il y a lieu de faire remarquer que les diverses nomenclatures qui ont été faites jusqu'ici des sections ou tribus Touareg sont devenues fausses, et qu'une nouvelle le deviendrait à bref délai. Dans cet état d'anarchie féodale et nomade, où vivent les Touaregs, avec leurs moeurs spéciales et leurs luttes incessantes, il se produit de continuelles modifications dans l'état des tribus. Sans cesse on constate des déplacements de sections ou de familles allant à la recherche d'une région où elles soient plus à leur aise ou mieux protégées./../ Dans ces conditions, il est inutile de rechercher trop exactement la nomenclature de ces collectivités changeantes, et il est préférable de retracer les traits principaux de l'état actuel du pays." (Cauvet $1903: 35)^{7}$

\footnotetext{
${ }^{6}$ Voir également les remarques de Rash à propos des Kel Owey (1973:29-30)

${ }^{7}$ Les difficultés rencontrées quand on essaye de cerner la segmentation propre aux tawsit n'est pas propre au monde touareg. On les retrouve notamment dans les études portant sur les tribus arabes. Exemple parmi d'autres ces lignes de R. Montagne à propos des Semmâr du Negd : "Établir un tableau complet et définitif de la structure d'une confédération bédouine est le plus souvent impossible/.../Si l'on parvient, en effet, à faire l'inventaire de toutes les sections et de tous les clans dont elle se compose, leur arrangement et la subordination réciproque des différentes divisions comportent en effet toujours un élément subjectif." (1932:65). Voir aussi Ashkenazi 1946.
} 
Nous n'avons échappé - faut-il le préciser - ni à ce problème ni à ces interrogations. Il n'est déjà point évident de cerner le phénomène de segmentation actuel mais, quand on essaye de le penser dans son rapport aux nombreuses nomenclatures déjà publiées, dans une démarche qui se veut diachronique et synchronique, l'entreprise devient fort ardue. Pour tenter d'éclaircir cette situation, plusieurs points doivent ici être précisés :

Il nous paraît que, dans les nomenclatures ci-dessus, n'a pas suffisamment été défini le niveau de segmentation où se situent les unités dénommées. D'où un certain nombre de confusions dommageables. La subdivision de la tawsit des Dag- $\gamma$ âli en plusieurs sections est un fait largement reconnu. Mais, entre ces dernières et l'unité domestique qu'est la tente, des niveaux intermédiaires peuvent encore être distingués. Tel est le cas du campement qui constitue, lui, l'unité résidentielle de base. Les membres d'un campement sont fréquemment désignés par référence soit au lieu géographique dans lequel ils ont l'habitude de résider, soit à l'homme considéré comme étant à l'origine de cette unité agnatique. Il existe là un niveau de définition qui, inférieur à la tawsit et à la section, est parfaitement opératoire chez les Kel-Ahaggar. Et l'on peut supposer que dans certaines des nomenclatures ci-dessus citées ce niveau de segmentation a parfois été confondu avec celui supérieur de la section ou de la tawsit. Tel nous paraît être le cas de ces Kel-Tesaylit ou de ces Kel-Tahat évoqués respectivement par Rognon et Lhote. Ces deux appellations renvoient à des zones géographiques connues. La première désigne la région basse qui borde sur son flanc sud-ouest la partie montagneuse de l'Atakor de Tamanrasset jusqu'à In-Amgel. La seconde désigne elle le plus haut sommet $(2918 \mathrm{~m})$ du massif de l'Atakor. Le groupe de personnes qui avaient l'habitude de résider dans le premier lieu étaient appelés Kel-Tesaylit. Ils ne constituaient pas une section autonome mais se rattachaient aux Kel-Tamanrasset. Aujourd'hui, comme tous les membres de cette section, ils sont sédentarisés à Tagmart. Mais, il est vrai qu'est encore utilisée l'appellation de Kel-Tesaylit (alors qu'ils ont quitté cette région depuis de nombreuses années déjà) quand on veut à l'intérieur même des Kel-Tamanrasset les distinguer des autres membres de la section. Quant à l'appellation Kel-Tahat, elle désigne, parmi les Kel-Tinhart, une lignée (en l'occurrence celle des descendants de Mohammed ag Ihemma ag Ider) qui se trouve être "propriétaire" de cette montagne du Tahat. Ces deux dénominations renvoient donc à des niveaux inférieurs à la section de tawsit. Dans les deux cas, un certain nombre de familles à l'intérieur même d'une section sont distinguées par une appellation faisant référence soit à leur lieu de résidence habituel (Kel-Tesaylit) soit à une zone territoriale sur laquelle ils bénéficient d'un droit de propriété (Kel-Tahat).

Un problème similaire se pose avec les Ulad-I $\gamma$ dal (= fils de I $\gamma$ dal)

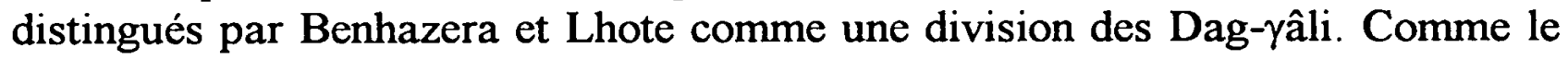
manifeste l'appellation utilisée, ce groupe de personnes se définit à partir d'un 


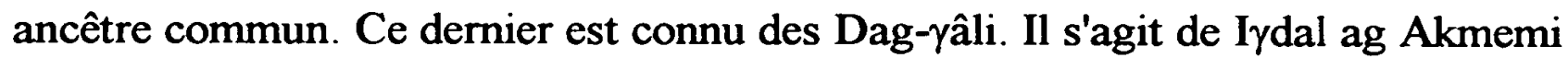
qui, notamment par l'intermédiaire de son fils Buhen, marié à Tamusat ult Hatita, fut à l'origine d'une nombreuse descendance. Mais cette dernière fait partie des Kel-Tamanrasset et n'a jamais, aux dires des Dag- $\gamma$ âli, constitué un sous-groupe autonome. Il y aurait donc là une confusion entre une section et un groupe de descendance et peut-être, au début du siècle, de résidence. Cet exemple des Ulad-I $\gamma$ dal pose d'ailleurs un autre problème. Nous avons précédemment évoqué l'impôt en nature (tiwsé) dû par les groupes tributaires à l'amenukal. Mais il arrivait assez fréquemment que ce dernier abandonne à des aristocrates démunis la perception de la part de tiwsé que lui devaient certains lignages tributaires. Tel était le cas des Ulad-I $\gamma d a l$. Ces derniers ne représentaient au début du siècle que 4 ou 5 tentes et payaient la tiwsé non à l'amenukal mais aux Kel-Emoyri des Inemba ${ }^{8}$ (cf. Benhazera 1908:146). On peut dès lors se demander si certains des groupes apparaissant dans les nomenclatures ci-dessus ne se singularisent pas simplement par des particularités dans le don de la tiwsé, ou par une relation spécifique appelée temazlayt. ${ }^{9}$

A cela viennent s'ajouter les confusions dues à la diversité et à l'évolution des termes utilisés pour désigner les différents segments. Ainsi voit-on apparaître chez Lhote des Dag-Mertemek ignorés de Benhazera alors qu'au contraire les

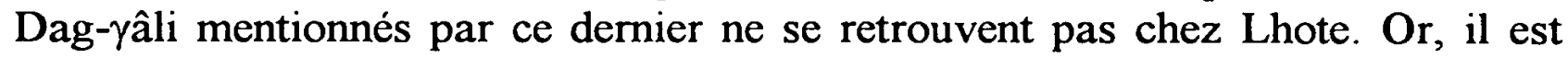
plus que probable, si l'on se réfère tant aux dires des Dag- $\gamma a ̂ l i$ qu'aux écrits du père de Foucauld, que ces deux appellations sont synonymes et désignent les mêmes personnes. La première étant anciennement utilisée, bien que de manière

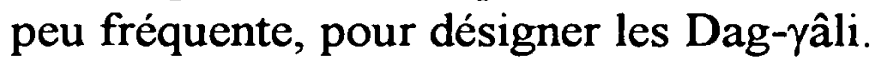

Toutes ces remarques n'annulent pas l'impression première que laisse la lecture de ces diverses nomenclatures. Nous pensons cependant qu'il faut aller au delà de cette constatation initiale et voir dans cette "confusion" un signe à interpréter, signe qui nous renseigne sur la nature même des tawsit. Les informations ci-dessus proviennent d'époques différentes : début du siècle pour Benhazera, 1940/1950 pour Lhote et 1960 pour Nicolaisen. Aussi serait-il erroné de déclarer véridique telle version et du même coup d'invalider les autres. De fait, ces nomenclatures sont les traductions plus ou moins fidèles de moments

\footnotetext{
${ }^{8}$ Tribu noble aujourd'hui agrégée aux Kel- $\gamma$ ela.

${ }^{9}$ Ce contrat de protection désigné par le terme temazlaït ("part spéciale") s'établissait entre des aristocrates et des tributaires appartenant ou non au même ettebel. De la part des premiers ce rapport impliquait un devoir de défense des lignages tributaires avec lesquels une relation de ce type s'était établie; en contrepartie, les tributaires se devaient eux de subvenir aux besoins de leur protecteur: redevances diverses, "cadeaux" plus ou moins imposés, prêts d'animaux, installation de la famille aristocratique auprès des campements tributaires qui se voient alors dans l'obligation de la "nourrir"...etc. Sur cette relation particulière, voir Gast 1972 et Keenan 1976.
} 
historiques différents et ce à propos d'une société en évolution permanente. Certes, de l'une à l'autre de ces listes, nombre et dénomination des sections varient, certaines apparaissent, d'autres disparaissent... Mais la densité et les articulations changeantes des sectionnements révèlent surtout le caractère dynamique d'un processus où fusion et fission se succèdent. Si erreur il y a, ce serait de croire de vouloir figer en un tableau statique une société en perpétuel mouvement. Loin d'être des cadres fixés de toute éternité, pratiquement inamovibles, les tawsit et leurs divisions internes sont sans cesse remodelées au gré des événements historiques dans lesquels entrent en compte - parfois de manière concomitante - des raisons d'ordre politique, démographique, économique, etc. C'est là, nous semble-t-il, une des objections majeures que l'on peut adresser à la théorie segmentaire. Celle-ci, d'Evans-Pritchard à Gellner, nous parait fondée sur un "oubli" de l'histoire, elle "condamne tous les segments à l'harmonie préétablie d'une sorte de présent enchanté " (Yacine 1990:45).

Or, tant au Maghreb (cf. Hammoudi 1974) que dans le monde touareg, la segmentation observable dans nombre de groupes est aussi - voire même surtout - produit de l'histoire et en ce sens sujette à perpétuelle évolution ${ }^{10}$.

\section{L'EXEMPLE DES Ikeccemâden ${ }^{11}$}

Pour illustrer notre propos, nous prendrons ici l'exemple des Ikeccemâden. Ceux-ci selon les époques et les auteurs ont été présentés soit comme un groupe

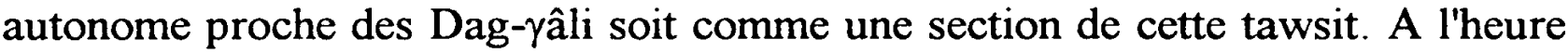

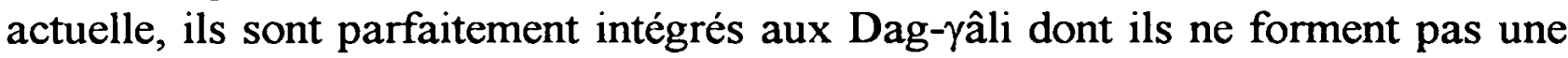
section particulière. Tout au plus rappelle-t-on parfois l'origine Ikeccemâden de tel ou tel individu, de telle ou telle famille. L'histoire des Ikeccemâden telle qu'elle est aujourd'hui narrée par les Dag-yâli nous paraît fort instructive dans la mesure où elle permet d'un peu mieux cerner les différents facteurs pouvant intervenir dans un processus de ce type. A cet effet, nous reproduisons cidessous le récit qui explicite et la naissance des Ikeccemâden et l'origine de leur appellation.

"Deux soeurs des Imesîliten avaient chacune un fils. A cette époque, une très forte famine régnait dans l'Ahaggar. Il n'y avait plus rien à manger. Aussi à tour de rôle chacune des soeurs partait à la recherche de plantes et de graines. Elles cueillaient des jujubes. Elles prenaient garde de les

\footnotetext{
${ }^{10}$ Voir également la critique portée par Berque (1978) à la théorie segmentaire dans le chapitre "Perspectives théoriques" ajouté, à la seconde édition, de son travail sur les Seksawa du Maroc. ${ }^{11}$ Les périodes de disette étaient fréquentes en Ahaggar et ce jusqu'à une époque récente ( $c f$. Galan 1951 et Malaurie 1953). D'où la nécessité d'une alimentation appropriée : récolte de plantes, racines, graines, etc (Gast 1968:195-262). En de telles circonstances, les fruits du jujubier (Ziziphus saharae (Batt.) Maire) dénommés ibekaten (sg. abaka) étaient particulièrement appréciés par les nomades Kel-Ahaggar.
} 
répartir équitablement entre ces deux enfants, entre fils et neveu. Un jour, poussée par la faim, une des soeurs s'en alla plus loin que d'habitude pour trouver de quoi manger. Elle atteignit ainsi un lieu nommé Ennedid. Elle ne connaissait point cet endroit et n'en avait jamais entendu parler. Là, elle trouva des palmiers, arbres qu'elle n'avait jamais vus auparavant et dont elle apprécia les fruits. Mais, jalouse de sa découverte, elle ne donna des dattes qu'à son seul fils enfreignant ainsi le partage égalitaire qui existait jusqu'alors. Devant cette injustice, l'autre enfant (son neveu) se mit à pleurer, puis alla se plaindre à sa mère. Celle-ci entra alors dans une forte colère et se fâcha avec sa soeur. Elle l'accusa de privilégier, de favoriser (= teshkeshemaded) son fils aux dépens de son neveu. C'est le terme employé en langue tamâhaq pour dénoncer ce favoritisme qui aurait donné naissance à l'appellation Ikeccemâden par laquelle furent désormais désignés les descendants de cette soeur indigne."

Outre l'hypothèse étymologique avancée ${ }^{12}$, ce récit justifie l'appropriation (sous forme collective) des palmiers d'Ennedid par les Ikeccemâden. Ce lieu est présenté comme une terre sans propriétaire située hors du territoire habituel des Imesîliten. Sa "découverte" par l'ancêtre des Ikeccemâden entérine l'actuel droit de propriété de ses descendants sur cette palmeraie ${ }^{13}$.

Mais il nous propose aussi d'autres enseignements plus fondamentaux quant à notre propos, et quant à l'origine des Ikeccemâden. Ceux-ci sont présentés comme issus d'une scission des Imesîliten. Par cette appellation est désignée l'important groupe tributaire dont seraient issus, si l'on s'en réfère à Benhazera (1908 : 107), les Dag- $\gamma a ̂$ li et les Kel-Ahnet ${ }^{14}$. De nos jours, le même terme désigne comme nous l'avons vu une des "moitiés" de la tawsit Dag-yâli. C'est parmi elle que se trouvent encore, comme l'avait déjà justement relevé Maitre (1977:773), des individus se reconnaissant comme de lointains descendants des Isabaten.

Deux points du récit ci-dessus nous paraissent fort révélateurs :

\footnotetext{
12 Nous n'avons point trouvé teshkeshemaded dans le Dictionnaire du Père de Foucauld. A moins (?) que ce terme se rattache à la série echchem-chouchem-achouchem (1951:128-130). Nos informateurs nous ont souvent proposé comme possible synonyme timuda, expression fréquemment employée quand - par jeu, le plus souvent - on manifeste une préférence pour une personne (souvent un enfant) et qu'on la privilégie par rapport aux autres.

${ }^{13}$ Ennedid est un petit centre de cultures situé sur les berges de l'oued Outoul à dix kilomètres en aval de Tin Amensar et à une douzaine de kilomètres du centre plus important d'Abalessa. Comme la plupart des autres centres de l'Ahaggar, Ennedid a connu une alternance de périodes d'abandon et de mises en cultures (cf. Pandolfi 1993 : 32). Se trouvent là une centaine de palmiers dont on n'a jamais pu déterminer l'origine.

${ }^{4}$ Foucauld ajoute lui que des descendants des Imesîliten seraient également intégrés aux AytLoayen (1940:246).
} 
- La scission qui provoquera la naissance des Ikeccemâden est exposée ici dans le langage de la parenté. Ce ne sont pas deux personnes quelconques qui se séparent mais deux soeurs. Ce recours à un schème parental est un phénomène fréquent chez les Kel-Ahaggar et dans le monde touareg en général. Est ainsi confirmée la (re)présentation que les tawsit se donnent d'elles-mêmes en tant que groupes de parents. De plus, sont ainsi justifiés les liens privilégiés pouvant exister entre deux groupes aujourd'hui autonomes mais hier unis par une relation (réelle ou supposée) de parenté. Ainsi les liens étroits qui ont longtemps existé

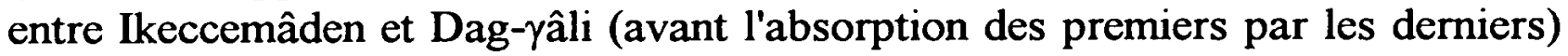
est elle présentée comme la manifestation d'une relation "originelle" de parenté entre les deux groupes.

-Dans ce cadre, on relèvera que ce sont deux personnages féminins (en l'occurrence deux soeurs) qui sont à l'origine de cette scission. C'est donc à une ancêtre féminine que se rattachent en dernière instance les Ikeccemâden. C'est là un "classique" du monde touareg dans la mesure où, comme le révèle également les récits concernant Tin-Hinan, c'est quasi-systématiquement à une fondatrice féminine qu'est rapportée l'origine des groupes. Ici ce trait se manifeste par le fait que les deux soeurs sont présentées comme des femmes sans hommes. Aucune mention n'est faite notamment des pères de leurs fils. Mais apparaît également que le principe fondateur féminin s'incarnera dans une descendance issue de ces personnages masculins que sont les fils. Si le principe féminin est en quelque sorte "originel" il se manifeste ensuite par l'intermédiaire de personnages masculins ${ }^{15}$.

Reste que dans le cas ici envisagé, l'hypothèse "parentale" proposée demeure largement insuffisante. La scission entre les deux soeurs ne peut à elle seule expliquer que les Ikeccemâden aient pu à un moment donné se constituer en tawsit. Aussi est-il nécessaire de faire intervenir l'efficience d'un cadre sociopolitique plus large, celui de l'ettebel Kel-Ahaggar.

Au milieu du XVIIIème siècle, lorsque l'amenukal Sidi ag Mokhammed elKheir procéda à une nouvelle répartition des groupes tributaires, les Imesîliten

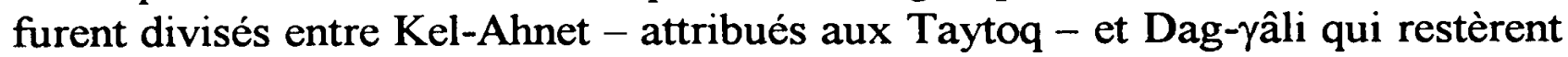
avec les Kel- $\gamma$ ela. Mais une partie des derniers nommés, en l'occurence les Ikeccemâden furent eux rattachés à l'ettebel des Taytoq. C'est à ces derniers qu'ils payèrent dès lors la tiwsé et ce jusqu'en 1907, quand le capitaine Dinaux décida que les Ikeccemâden seraient désormais comptés avec les Dag-râli et payeraient la tiwsé avec eux. Cette décision de Dinaux est certes la conséquence logique d'un constat évident. Bien que faisant partie de l'ettebel des Taytoq les

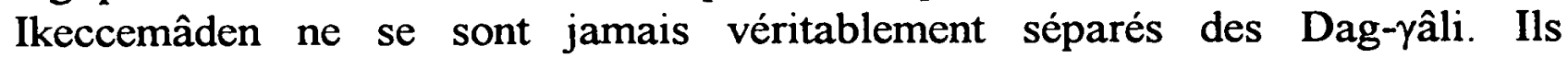

\footnotetext{
${ }^{15}$ Schème que l'on trouve parfaitement illustré dans la transmission du pouvoir tant chez les aristocrates Kel- $\gamma$ ela au niveau de l'ettebel que chez les tributaires Dag- $\gamma a \hat{l i}$ au niveau de la tawsit : voir Pandolfi 1995a.
} 
continuaient à vivre à leurs côtés et de nombreuses alliances réactivaient les liens existant entre ces deux groupes. Mais, la décision de Dinaux tend aussi à affaiblir les Taytoq alors considérés comme des éléments instables et peu coopératifs et, au contraire, à renforcer les Kel- $\gamma$ ela et Musa ag Amastan ${ }^{16}$.

Mais ce qui nous importe ici est bien que la constitution des Ikeccemâden en tant que tawsit est avant tout le résultat d'une décision politique prise au niveau de l'ettebel. Décision qui constitue les descendants de la "soeur indigne" en groupe autonome par leur rattachement aux Taytoq et les distingue des Dag-

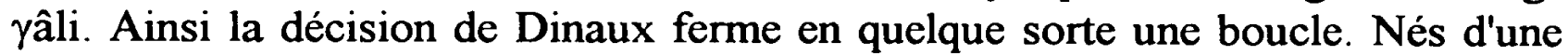
scission des Imesîliten, les Ikeccemâden après une époque d'autonomie se retrouvent de fait réintégrés aux Dag- $\gamma a ̂ l i$. Ils semblent alors avoir constitué durant un certain temps, si l'on s'en réfère aux nomenclatures de Demoulin et Lhote, une des sections de cette tawsit. Aujourd'hui ils se retrouvent complètement intégrés aux Dag- $\gamma a ̂ l i$ dont ils ne représentent même plus une section. Soit le schéma suivant :

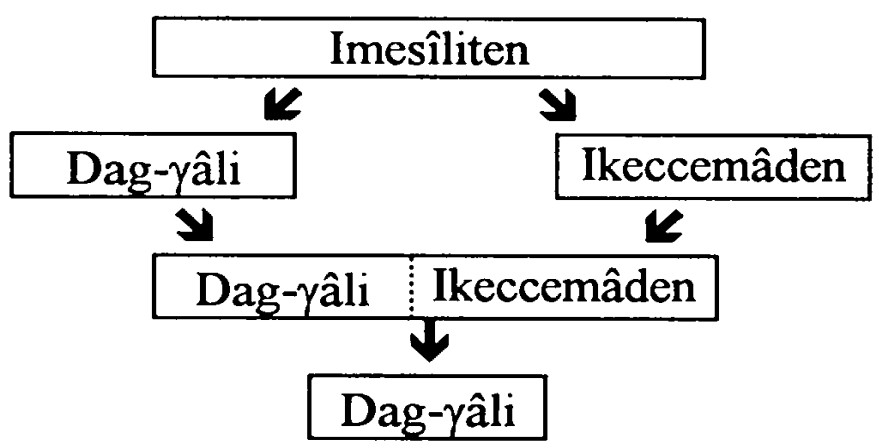

L'exemple Ikeccemâden révèle ainsi quelques uns des points fondamentaux (scission/fusion, alliances matrimoniales, efficience du cadre politique global...) qui sont à l'oeuvre tant dans l'apparition que dans la disparition, en tant que groupe autonome, d'une tawsit. On n'oubliera pas cependant de relever l'importance déterminante du facteur démographique. La richesse et la force d'une tawsit sont aussi - voire surtout - dépendantes de son poids démographique. Dans le cas des Ikeccemâden, tout indique qu'à aucun moment ils n'ont pu constituer un groupe d'importance à ce niveau. Nombre d'auteurs ont au contraire relevé, à des époques différentes, leur faiblesse démographique (Benhazera 1908, Lhote 1955 et Keenan 1977 notamment). Or, dans l'ensemble du monde touareg, une faiblesse démographique, quelles qu'en soient ses causes, est bien souvent une caractéristique commune aux groupes qui ont disparu en

\footnotetext{
${ }^{16}$ Les Ikeccemâden ne furent pas le seul groupe tributaire transféré de l'ettebel des Taytoq à celui des Kel- $\gamma$ ela. Tel fut également le cas des Ikuttisen. A propos de cette décision, le capitaine Dinaux se montre on ne peut plus clair : "...étant donné le parcours normal de cette fraction, l'impuissance de Sidi ag Keradji à la commander et l'intérêt que nous avons à augmenter l'influence de Moussa, la fraction des Ikoutisen est provisoirement rattachée à l'aménokalat du Ahaggar." (AOM 22H73). Voir Pandolfi 1993.
} 
tant que tels et qui ont vu leurs membres intégrés dans des tawsit plus importantes.

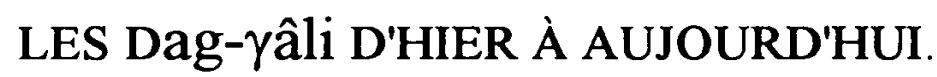

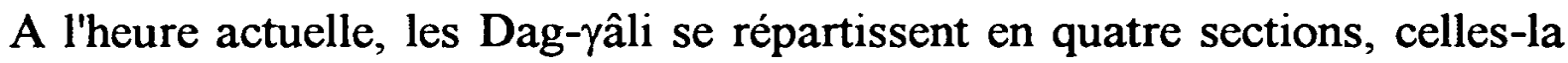
même que donnait déjà Nicolaisen, à savoir : Kel-Terhenânet, Kel-Tamanrasset,

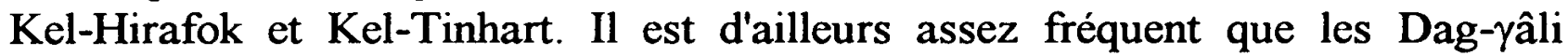
comparent leur tawsit à un chameau dont les quatre pattes seraient les quatre sections ci-dessus. De plus, suite à la sédentarisation des Dag- $\gamma$ âli, cette segmentation s'est en quelque sorte concrétisée au niveau résidentiel. De nos

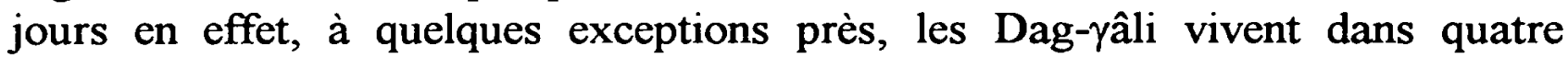
villages. Chacun d'eux est situé dans l'aire de nomadisation habituellement dévolue à chacune des sections ci-dessus. Et les membres masculins de chacune d'entre elles se retrouvent de fait résidents du même village.

Mais si cette segmentation en quatre sections (devenues aussi de fait des groupes résidentiels) ne pose guère problème, il n'en est point de même de la bipartition supérieure avancée par Nicolaisen. Celui-ci estime que la tawsit des

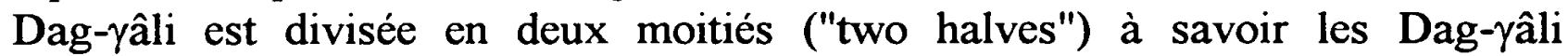

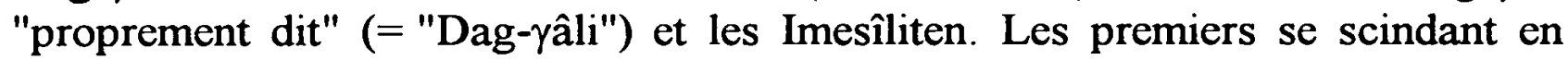
Kel-Tamanrasset et Kel-Terhenânet, les seconds en Kel-Hirafok et Kel-Tinhart.

"Some tuareg of the Dag-Rali may claim that these two halves are matrilineally related to one another, but also that they do not descend from sisters. It seems in fact as if these two halves stand in a cross-cousin relation ship to one another, and it is agreement with this that the Dag-Rali proper speak of the Imesilliten as "children of their mother's brother". The two halves in the past intermarried much though marriages between them are very rare nowadays." (1963:140).

Cette présentation, également proposée par Bourgeot (1972:542), paraît

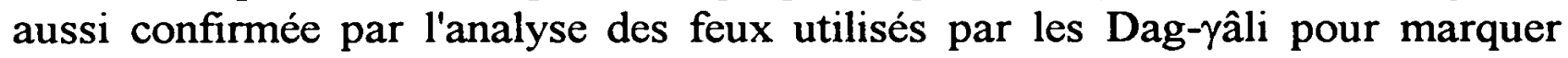
leurs camelins. Dans cette tawsit, les chameaux sont marqués au fer rouge sur le côté droit du cou. Les marques utilisées sont fort nombreuses et variées. Elles indiquent tout à la fois le groupe de descendance, la section et la famille auxquelles se rattachent le propriétaire de l'animal. Quelques grands traits communs se dégagent cependant.

Ainsi, tous ces chameaux portent une barre verticale $\mid{ }^{17}$ qui indique leur

${ }_{17}$ Pour Bourgeot (1972:549) cette barre verticale commune à toutes les marques Dag-râli pourrait laisser "supposer l'existence ancienne, mais périmée, d'une appropriation collective du troupeau au bénéfice de l'ensemble des membres à part entière du lignage." C'est là une hypothèse séduisante mais aucun élément autre ne permet cependant de véritablement la conforter. 


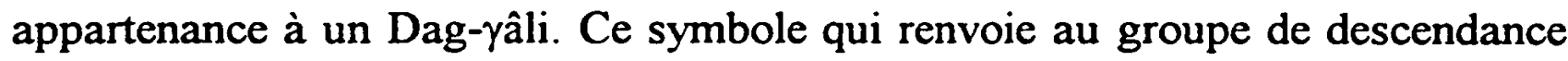
se combine avec d'autres éléments qui indiquent, eux, la section. Soit :

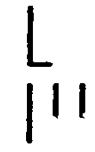
Kel Hirafok
$F$
Kel Tinhart.
Kel Terhenanet

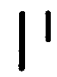
Kel Tamanrasset

Ce type de feu qui indique donc et la tawsit et la section est dénommé éhwel en tamâhaq (Foucauld 1951:629). Il est particulièrement significatif que les symboles utilisés reprennent la bipartition relevée par Nicolaisen. Les Imesîliten combinent la marque de la tawsit avec un trait horizontal alors que les "Dag-râli" associent la marque commune à tous les membres du groupe de descendance avec un (Kel Tamanrasset) ou deux petits traits verticaux (Kel Terhenanet). A ces marques viennent s'ajouter des feux particuliers qui se transmettent par voie patrilinéaire. Ces marques "individuelles" sont, elles, dénommées azezlu en tamâhaq (Foucauld 1951:1965). La combinaison éhwel + azezlu donne alors des feux de ce type :

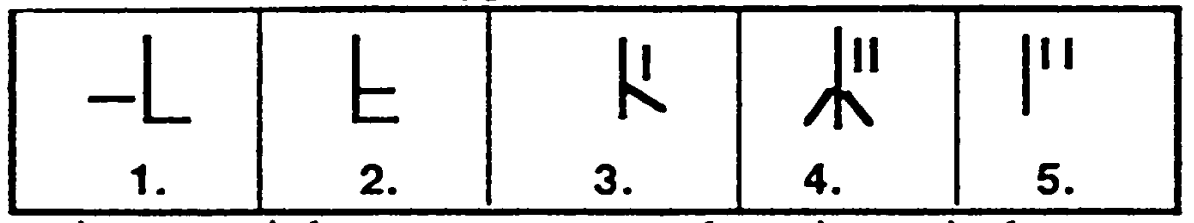

Dans les cinq cas ci-dessus se retrouve le trait vertical propre à la tawsit Dag-yâli. Dans les exemples (1) et (2) les animaux appartiennent à des KelHirafok, dans les exemples (3) et (4) à des Kel Tamanrasset et enfin dans l'exemple (5) à des Kel Terhenanet.

Lors de nos enquêtes, cette affirmation d'une bipartition de la tawsit Dagyâli nous a longtemps posé problème et ce d'autant plus qu'aucun de nos informateurs ne se montrait capable de nous apporter quelque lumière sur ce sujet. Tout au plus obtenions-nous quelques brèves affirmations qui, bien souvent, nous paraissaient contradictoires les unes avec les autres. Aussi, nous avons abandonné un certain temps l'idée d'un tant soit peu élucider cette question. Ce n'est qu'après avoir recueilli un assez grand nombre de généalogies qu'il nous parut temps d'y revenir en confrontant ce que nous avions pu apprendre de l'histoire de cette tawsit aux données généalogiques. Si les résultats ainsi obtenus doivent être présentés avec la prudence qu'impose ce type d'enquête, nous pensons néanmoins qu'ils traduisent le seuil de connaissances auquel on peut prétendre. Il est malheureusement vraisemblable que l'on ne puisse guère aller au-delà vu la rapidité avec laquelle la mémoire de cette tawsit disparaît au rythme des décès qui emportent les quelques rares personnes âgées qui en sont encore les dépositaires.

Une des difficultés majeures à laquelle nous nous sommes heurté provenait du nom même et de la tawsit, et d'une des moitiés repérées par Nicolaisen. Nous avons, en effet, vainement cherché dans un premier temps à retrouver le schéma proposé $(\mathrm{Dag}-\gamma \hat{\mathrm{l}} \mathrm{l}$ = fils de $\gamma$ âli) par cette appellation. Or, si l'ancêtre éponyme 
joue certes un rôle important, il est un autre personnage fondamental, en l'occurence son épouse Debeinnu ult Awata. On ne peut appréhender ni l'histoire ni la structure de ce groupe tributaire sans la prise en compte de ce couple et de leurs enfants. Il nous faut donc ici, à l'aide des renseignements recueillis, présenter brièvement l'histoire de cette tawsit telle qu'elle est proposée par les intéressés eux-mêmes dans sa trame parentale ( $c f$. Fig. 1).

Figure 1.

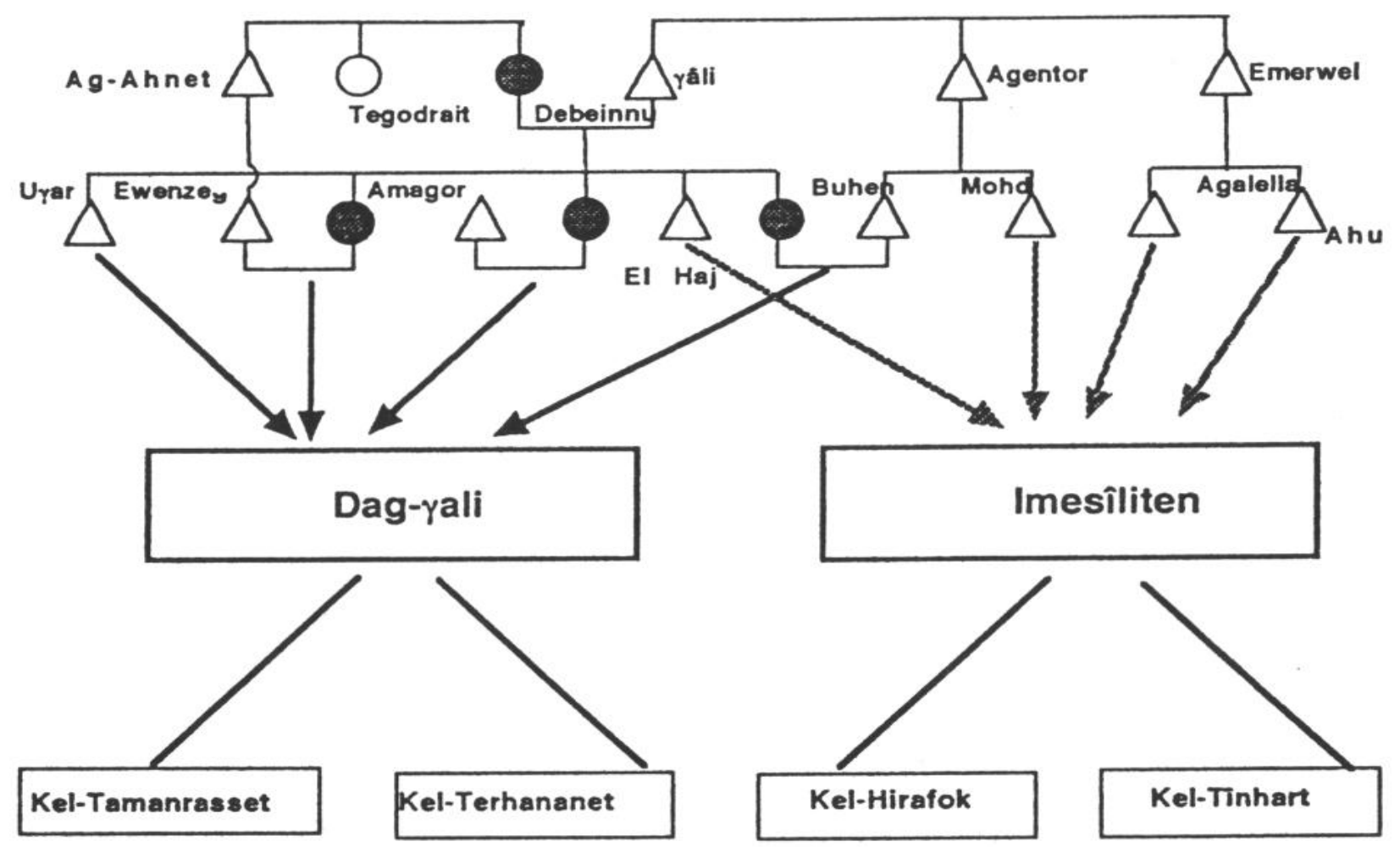

yâli avait deux frères cadets : Agentor et Emerwel ${ }^{18}$. Debeinnu, elle, aurait eu au moins un frère aîné (Ag-Ahnet) et une soeur cadette (Tegodrait). Les deux frères de $\gamma$ âli et $\mathrm{Ag}$-Ahnet sont à l'origine de lignées dont on retrouve nombre de descendants parmi les Dag-yâli actuels. Mais, pour aucun des trois, n'a pu être précisé le nom de leurs épouses.

${ }^{18}$ Les propos et récits recueillis chez les Dag-yâli laissent présumer qu'Agentor était l'aîné d'Emerwel. Lors de notre dernier séjour dans l'Ahaggar nous nous sommes rendus sur les tombes présumées être celles des divers personnages évoqués ci-dessus. Celle dEmerwel se trouve dans l'oued Hirafok au lieu-dit "celui du figuier". A quelques quatre kilomètres de là, à la confluence de l'oued Hirafok et de l'oued Egig, se trouve celle dUr ay ag yâli, fils de son frère

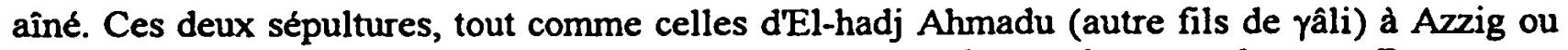
celle d'Ag-Ahnet (frère de Debeinnu) à Terhenânet, sont des tombes musulmanes. Par contre, celle de yâli lui-même, située dans l'oued Ellel au Nord de l'Assekrem, se présente sous la forme d'un tombeau pré-islamique (edebni). 
Du couple $\gamma$ âli/Debeinnu seraient issus six enfants : trois garçons (Ur a $\gamma, \mathrm{El}$ haj Ahmadu et Tirtin) et trois filles (Ettegaget, Tahart et Tederfut).

Les mariages de ces dernières sont connus et nous ont été confirmés par plusieurs informateurs :

- Ettagaget a épousé son cousin parallèle patrilatéral Buhen ag Agentor.

- Tahart, son cousin croisé matrilatéral, Ewenzeg ag Ag-Ahnet.

- Tederfut, un "étranger" venu des Ajjers, Amagor ag Akotey.

En ce qui concerne les fils de yâli, les renseignements sont plus fragmentaires et plus confus. L'un d'eux dont le nom (Tirtin) s'est conservé jusqu'à aujourd'hui ne semble avoir eu aucune descendance.

Tel n'est point le cas du fils âné, Ur ay ag yâli, dont les descendants ont joué un rôle important dans l'histoire de la tawsit. Mais, si les "enfants" d'Ur a sont bien connus, on ignore qui était son épouse. Enfin, El hadj Hamadu a eu une très nombreuse descendance issue de quatre voire cinq mariages.

Tous les personnages ci-dessus sont à l'origine des principales lignées ${ }^{19}$ constituant la tawsit. On peut remonter jusqu'à eux la généalogie de nombre de Dag- $\gamma$ âli. Mais, concernant la division "Dag- $\gamma$ ali" /Imesîliten, un point capital apparaît :

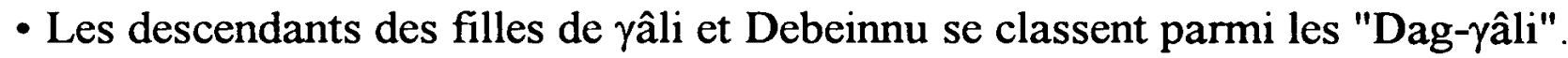
- Les descendants des fils des frères de yâli comme ceux d' El Hadj Ahmadu (fils de $\gamma$ âli) font, eux, partie des Imesîliten.

Cette différenciation apparaît de façon fort significative quand on envisage de manière plus précise ce qu'il advient des descendances d'Emerwel et Agentor, frères de $\gamma$ âli.

Du premier, on connaît deux fils dénommés Agalella et Ahu dont les descendants font partie des Imesîliten. Le second eut lui aussi deux fils : Mohammed et Buhen. Or, si logiquement les descendants de Mohammed font également partie des Imesîliten, tel n'est point le cas de ceux de Buhen qui

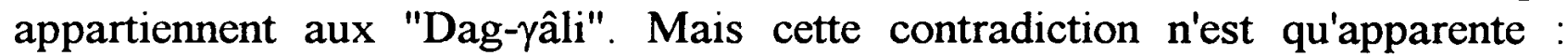

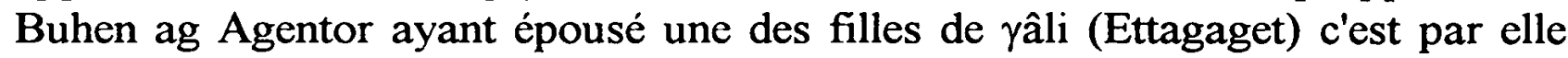

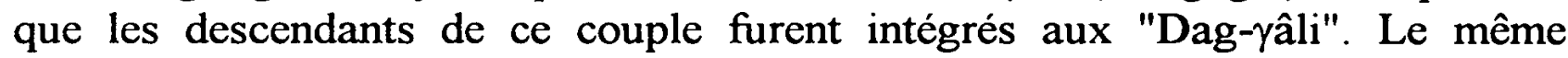
phénomène s'observe aussi pour les fils d'Ag-Ahnet, leurs descendants sont des

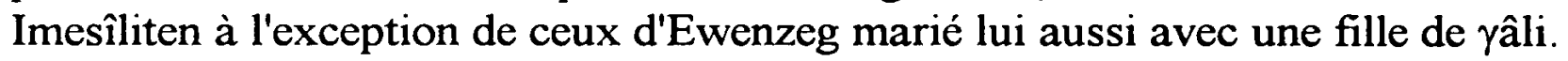

Dans cette optique, il demeure cependant un problème posé par la descendance d'Ur a $\gamma$ ag $\gamma$ âli. En effet les descendants de ce dernier, à la

19 Dans la littérature ethnologique, outre l'accentuation du caractère unilinéaire, le terme lignage sous-entend que l'on se trouve en présence d'une unité exogame. Pour cette raison majeure, il ne nous paraît pas approprié d'employer ce terme dans le cas des Kel-Ahaggar qui recherchent et pratiquent des alliances au plus près, parmi les parents proches. D'où, ce pisaller qui nous fait ici parler de lignées (terme moins connoté à nos yeux) pour désigner les membres qui, à l'intérieur même de la tawsit, se réclament d'une ascendance commune. 
différence de ceux d'El hadj Ahmadu son cadet, appartiennent sans contestation possible à la moitié "Dag-yâli " (section Kel-Tamanrasset). Ils comptent parmi les plus influentes familles de la tawsit à qui ils ont fourni quelques-uns de ses chefs (amyar). Problème d'autant plus difficile à résoudre qu'on ne connait pas l'épouse d'Ur a $\gamma$ ag $\gamma$ âli. Plusieurs noms nous ont été proposés mais aucun ne fait l'unanimité et n'apparaît certain. On relèvera cependant, parmi ces diverses propositions, qu'il pourrait s'agir d'une fille de Tegodrait, soeur cadette de Debeinnu ult Awata.

Fréquemment les Dag- $\gamma a ̂ l i$ explicitent la division de leur groupe de descendance en présentant les Imesîliten comme les descendants en voie

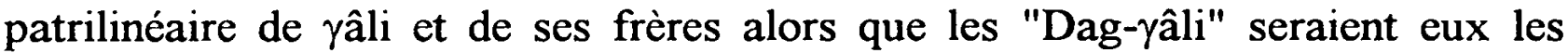
descendants en voie matrilinéaire de Debeinnu et de ses filles. Pour justifier la bipartition de la tawsit il est alors fait référence aux notions de tesa et aruri. Ces termes désignent respectivement le "ventre" et le "dos". Mais, outre ces significations corporelles, tous deux sont aussi fréquemment utilisés pour ces deux axes d'identification majeurs que sont les chaînes parentales patri- ou matri-linéaire. Aruri désigne ainsi, par extension, "le côté paternel (côté duquel on descend par son père et ses aïeux paternels d'homme en homme; côté duquel on descend en lignée paternelle de père en fils)." (Foucauld 1951:1797). Le terme tesa désignant lui "...le côté maternel...le côté duquel on descend en lignée maternelle de mère en fille..." (Ibid.:1660). Dans le "discours légaliste de la parenté" ${ }^{20}$ tenu par les Kel-Ahaggar, il est systématiquement fait référence à un principe d'unilinéarité pour justifier les principales caractéristiques de la société (recrutement des unités sociales, transmission du pouvoir, héritage des biens, résidence, etc). Selon les cas envisagés, sera mis en avant soit l'axe matrilinéaire (tesa) et la chaîne des "mères" (mas en mas en ma) soit l'axe patrilinéaire (aruri) et la chaîne des "pères" (tis en ti en $t i$ ). Nombre de nos informateurs estiment

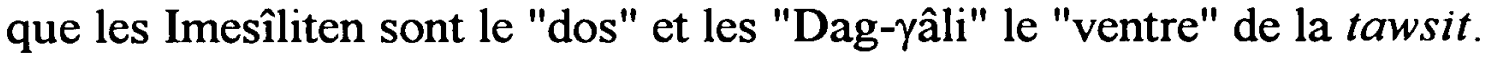

Présentation parfaitement justifiée par la trame parentale exposée dans la figure 2, ci-après. Il est d'ailleurs possible d'épurer ce schéma afin de mieux laisser apparaître que la bipartition de la tawsit Dag-yâli trouve son origine dans la relation frère/soeur et dans la différenciation sexuelle qui s'y inscrit.

Cette hypothèse permet également de rendre compte de certaines

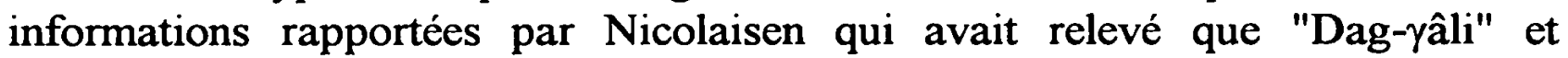
Imesîliten se trouvaient dans une relation de "cousins croisés" et que nombre de

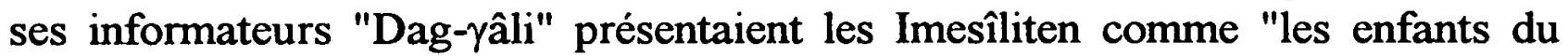
frère de notre mère" (1963:141). Si l'on rapporte ce type de propositions à la génération des descendants directs du couple yâli/Debeinnu, il paraît évident que

\footnotetext{
${ }^{20}$ Nous reprenons là une expression utilisée par H. Claudot en titre dun de ces articles sur la parenté touarègue (1983).
} 
la différenciation s'établit ici entre les fils et filles de ce couple, entre frères et soeurs.

FIgure 2.

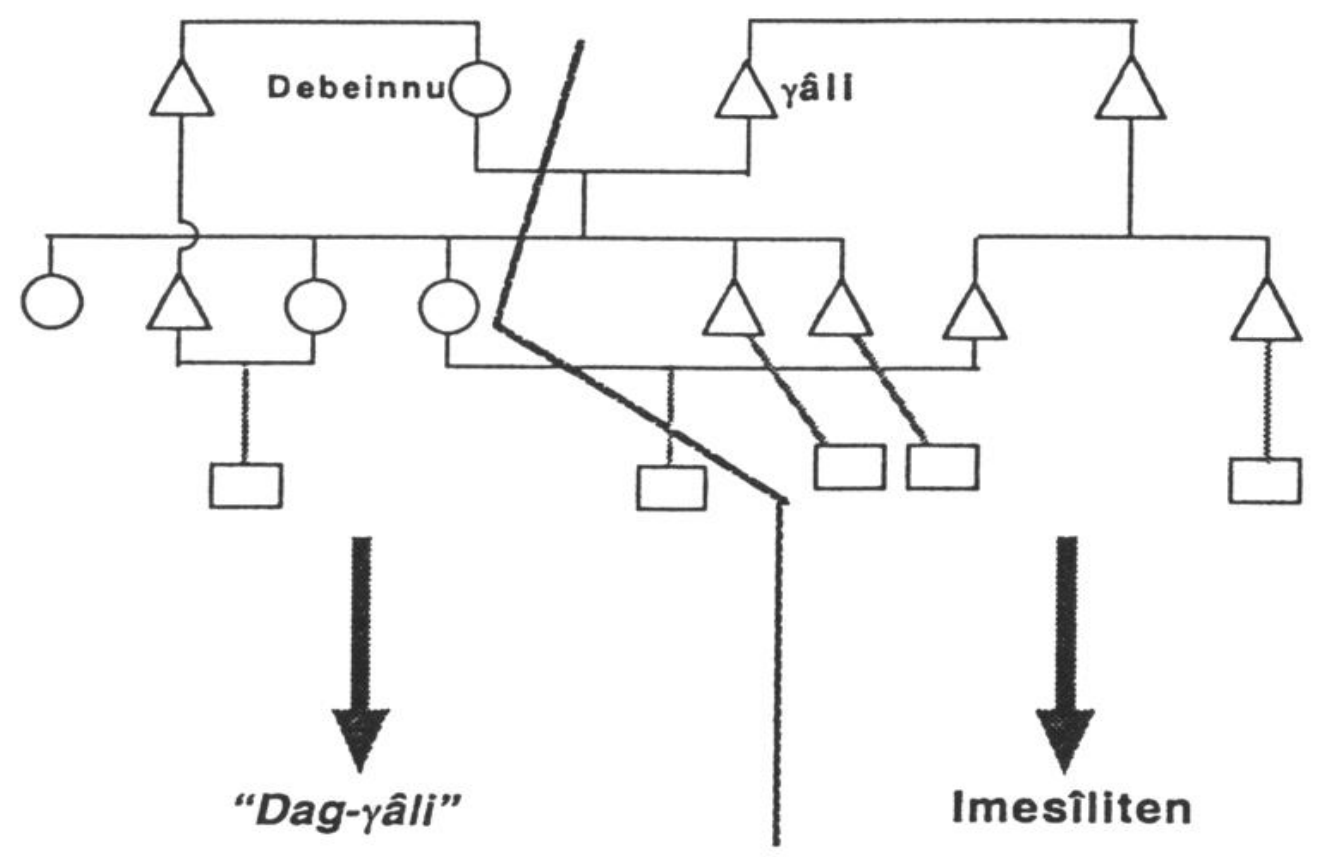

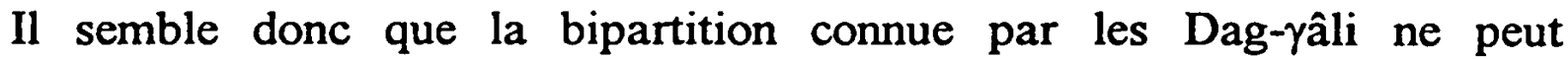
s'expliquer que si l'on prend en compte le critère de bifurcation. Les descendants

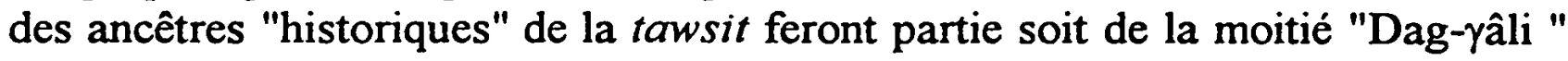
soit des Imesîliten selon qu'ils se trouvent reliés à yâli par des chaînons féminins ou par des chaînons masculins. Encore faut-il préciser que, dans le cas où ces deux possibilités coexistent, c'est l'élément féminin qui l'emporte comme nous l'avons vu par les exemples de Buhen ag Agentor et d'Ewenzeg ag Ag-Ahnet. De fait, si on accepte l'hypothèse ci-dessus évoquée quant à l'identité de la femme d'Ur ay ag yâli, le point capital est de pouvoir ou non se rattacher par leurs filles soit à Debeinnu soit à sa soeur cadette Tegodrait. Demeure cependant le problème posé par l'appellation même d'Imesîliten. Si l'on se réfêre au Père de Foucauld, ceux-ci

"étaient autrefois une tribu nombreuse ; aujourd'hui, ils n'existent plus à l'état de tribu; ils sont dispersés, fondus dans d'autres tribus et presqu'éteints, il ne subsiste d'eux que des familles isolées vivant la plupart avec les Dag-R'ali, quelques-unes avec les Ayt-Loayen, quelques-unes avec les Kel-Ahnet." (1940:246).

D'autre part, dans une version de la légende de Tin-Hinan recueillie par Benhazera, les Imesîliten sont présentés comme les descendants de Takama. Par la suite, une scission de ce groupe aurait donné naissance aux Dag-râli et aux 
Kel-Ahnet. Après avoir plusieurs fois signalé l'ancienneté des Imesîliten (1908:90), Benhazera précise que cette scission eut lieu lors de la création des ettebel Taytoq et Tégéhé-Mellet et du nouveau partage des groupes tributaires qui s'en suivit. L'amenukal Sidi "divisa en deux les Imesîliten. Il en donna une partie aux Taytoq (ce sont les Kel-Ahnet) et il garda le reste pour lui (ce sont les Dag-Rali)" (1908:107).

Ces diverses informations ne sont pas toujours concordantes. Benhazera

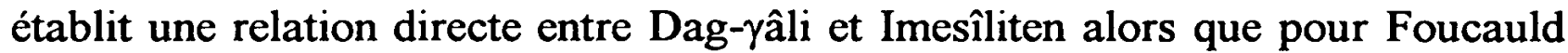
ces derniers ne représentent qu'une partie ("quelques familles") des Dag- $\gamma a ̂ l^{21}{ }^{21} \mathrm{De}$ nos jours, très peu de Dag-yâli peuvent encore apporter quelques renseignements sur un tel sujet. Le trait le plus fréquemment répété demeure l'affirmation que les Imesîliten sont à compter parmi les plus anciens occupants de l'Ahaggar. De plus, certains voient en l'ancêtre éponyme de leur tawsit un membre des Imesîliten. Ceci se conjugue d'ailleurs, et c'est là nous semble-t-il un point fort significatif, avec un refus, ou du moins une forte réticence, à voir en lui un descendant de Takama et ce à la différence de son épouse Debeinnu présentée elle comme "fille" de Takama.

A cet ensemble de données doit être ajouté un point déjà relevé par Maitre (1977:773) : les Dag- $\gamma$ âli qui se présentent comme lointains descendants des Isabaten appartiennent tous aux Imesîliten. C'est d'ailleurs parmi eux que se trouvent des individus qui ont des prérogatives sur certaines zones du territoire

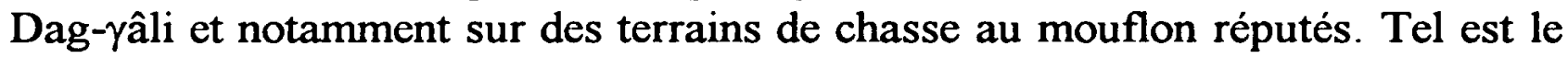
cas, entre autres, du mont Tahat attribué, par transmission patrilinéaire, aux descendants de Mahwa ag Ihema.

Autant d'indices qui permettent d'avancer avec prudence l'hypothèse suivante : dans la tawsit Dag-yâli, les Imesîliten relèvent d'une implantation ancienne voire sont, au moins pour certains d'entre eux, à relier aux Isabaten. Par contre, une arrivée plus récente serait symbolisée par Debeinnu présentée comme une "fille" de Takama.

L'union de Debeinnu avec yâli concrétise la fusion de ces divers groupes en

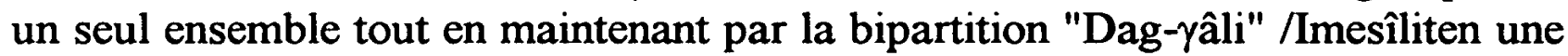
différenciation interne révélatrice. Cette bipartition est reconduite par les

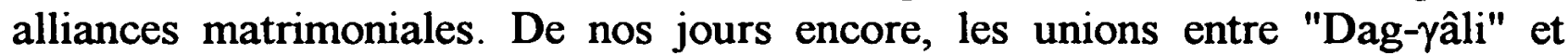
Imesîliten sont nettement moins nombreuses que celles internes à chacune des "moitiés" de la tawsit.

Les traits les plus marquants de cette division révèlent en ce qui concerne les Imesîliten une position d'infériorité hiérarchique. Ces derniers, en effet, se trouvent dans l'impossibilité d'accéder tant au pouvoir au niveau de la tawsit

\footnotetext{
${ }^{21}$ De même, Foucauld ne mentionne pas les Imesîliten dans les deux principales versions de la légende de Tin-Hinan qu'il a recueillies. Il ne les présente pas non plus, à la différence de Benhazera, comme les descendants de Takama.
} 
(ampar) qu'aux biens collectifs ettebel (cf. Pandolfi 1995 a et b). Par contre, la reconnaissance pour certains d'entre eux d'une "priorité" sur certaines parties du territoire de la tawsit recoupe l'affirmation de l'antériorité de leur implantation en ces lieux. Tableau qui n'est en rien contradictoire avec l'hypothèse précédemment avancée. Se marqueraient là, en effet, et l'ancienneté de l'implantation des Imesîliten et une arrivée (une invasion?) plus récente d'individus qui auraient pris le dessus sur eux et les auraient maintenus - tout en les intégrant dans leur groupe - en position d'inférioritée ${ }^{22}$.

\section{Références bibliographiques}

Archives d'Outre-Mer/Aix en Provence.

- Rapport de tournée du Capitaine Dinaux (03/05 au 29/10/1907), Carton 22 H 73.

- Tableaux de l'organisation du commandement des tribus, Série $8 \mathrm{HH}$.

ASHKENAZI, T. 1946-1949. "La tribu arabe : ses éléments », Anthropos XLI-XLIV : 657-672.

BENHAZERA, M. 1908. Six mois chez les Touaregs du Ahaggar, Alger, Jourdan, 234p.

BERQUE, J. 1978. Structures sociales du Haut-Atlas, ( ${ }^{\circ}$ éd. rev. et augm.), Paris, P.U.F., 513p

BERNUS, S. 1976. "Stratégie matrimoniale et conservation du pouvoir dans l'Aïr et chez les Iwllemmeden ", Revue de l'Occident Musulman et de la Méditerranée, $\mathrm{n}^{\circ} 21: 101.109$.

BERNUS, S., BONTE, P., BROCK, L., CLAUDOT, H. (éds) 1986. Le Fils et le neveu. Jeux et enjeux de la parenté touarègue, Cambridge et Paris, C.U.P. et M.S.H., 343p.

BONTE, P. 1986. "Introduction" in : S. Bernus \& al. (éds). Le Fils et le neveu : 3-36.

BOURGEOT, A. 1972. "Idéologie et appellations ethniques. L'exemple twareg. Analyse des catégories sociales ", Cahiers d'Etudes Africaines, vol. XII, 48 (4): 533-554.

CAMPS, G. 1986. «Préhistoire de l'Ahaggar », Encyclopédie berbère III : 275282.

CAUVET, G. 1903. "Notice sur les fractions touareg du sud d'In-Salah », Bulletin du Comité de l'Afrique Française 13 (1) : 35-38.

CLAUDOT, H. 1983. "Le discours légaliste de la parenté chez les Touaregs ", Annuaire de l'Afrique du Nord 22 : 533-543.

\footnotetext{
${ }^{22}$ On pense un peu à ces sociétés sahéliennes, où s'opposent des "maitres de la terre" et des "maîtres des gens". Voir, par exemple, Izard 1993 (ch.10) et Pouillon 1993:1125.
} 
DEMOULIN, F 1926. "Les Touareg du Hoggar», Bulletin du Comité de l'Afrique Française/ Renseignements Coloniaux 4 : 174-185.

FOUCAULD, Ch. de. 1940. Dictionnaire abrégé Touareg-Français des noms propres, (Dialecte de l'Ahaggar), Paris, Larose, 362p.

FOUCAULD, Ch. de. 1951. Dictionnaire touareg-français. Dialecte de l'Ahaggar, Paris, Imprimerie Nationale, 4 vol, 2028p.

GALAN, P. 1951. "Contribution à l'étude du problème alimentaire au Hoggar ", Archives de l'Institut Pasteur d'Algérie XXIX : 230-243.

GAST, M. 1968. Alimentation des populations de l'Ahaggar, Mémoires du CRAPE. VIII, Paris, A.M.G., 458p.

GAST, M. 1972. "Temazlayt (Contrat de protection chez les Kel-Ahaggar) ", Encyclopédie Berbère, édit. prov., Cahier ${ }^{\circ} 7 ., 2 \mathrm{p}$.

- 1988. "Anbiya ", Encyclopédie Berbère V : 635-636.

HAMMOUDI, A. 1974. "Segmentarité, stratification sociale, pouvoir politique et sainteté ». Réflexions sur les thèses de Gellner, Hesperis-Tamuda 15 : 147180.

IZARD, M., 1993, L'odyssée du pouvoir. Un royaume africain : Etat, soclété, destin individuel, Paris, EHESS, 156p.

KEENAN, J. 1976. "Some theoretical considerations on the "temazlayt" relationship ", Revue de l'Occident Musulman et de la Méditerranée 21 : 33-45.

- 1977. The Tuareg : People of Ahaggar, London, Allen Lane, 385p.

LHOTE, H. 1979. "Le vêtement de peau chez les Touaregs. Hypothèse de son origine ", Bull. Arch. Maroc. XII: 323-354.

- 1984. Les Touaregs du Hoggar, ( $1^{\circ}$ éd. 1944), Paris, A. Colin, 255p.

MAITRE, J.P. 1977. "Contribution à la préhistoire récente de l'Ahaggar dans son contexte saharien ", Bull. de l'I.F.A.N., 38, $\mathrm{B}, 4: 715-789$.

MALAURIE, J. 1953. "Touareg et Noirs au Hoggar, Aspects de la situation actuelle », Annales E.S.C. VIII : 338-346.

MONTAGNE, R. 1932. "Notes sur la vie sociale et politique de l'Arabie du Nord : Les Semmâr du Negd ", Revue des Etudes Islamiques.

NICOLAISEN, J. 1963. Ecology and Culture of pastoral Tuareg, Copenhague, National Museum, 548p.

PANDOLFI, P. 1993. "De l'origine d'un nom à l'histoire d'une tawsit " in : Le politique dans l'histoire touarègue (H.Claudot-Hawad éd.), Cahiers de l'IREMAN 4 : 25-34.

- 1995a. "Amghar $\mathrm{n}$ tawsit et ettebel : la transmission du pouvoir chez les Dag-Ghâli de l'Ahaggar ", Awal-Cahiers d'Études Berbères 12:99-121.

- 1995b. Le ventre et le dos. Parenté et résidence dans un groupe tributaire de l'Ahaggar: les Dag-yâli, Thèse d'ethnologie, Université de la Réunion, 616p. POUILLON, J., 1993, Le cru et le su, Paris, Editions du Seuil, 176p.

RASH, Y. 1973. Les premières années françaises au Damergou, Paris, Société Française d'Outre-Mer et Librairie P.Geuthner, 144p. 
ROGNON, P. 1962. "La confédération des nomades Kel-Ahaggar », Annales de Géographie 71 : 604-619.

TILLION, G. 1973. "Les deux versants de la parenté berbère » in : Actes du ler Congrés d'Etudes des Cultures méditerranéennes d'influence araboberbère : 43-49.

YACINE, T. 1990. Poésie berbère et identité, Alger, Bouchène-Awal, 444p. 